\title{
Nano-Coated Heat Pipe Plate for Miniaturized Electronics Cooling
}

\author{
Yanping $\mathrm{Du}^{1}$ and Yonggang $\mathrm{Zhu}^{2,3}$ \\ ${ }^{1}$ China-UK Low Carbon College, Shanghai Jiao Tong University \\ No.3 Yinlian Rd, Pudong district, Shanghai, China \\ Yanping.Du@sjtu.edu.cn; \\ ${ }^{2}$ Harbin Institute of Technology (Shenzhen) \\ Shenzhen, Guangdong 518055, China \\ ${ }^{3}$ School of Science, RMIT University \\ Melbourne, VIC 3001, Australia \\ zhuyonggang@hit.edu.cn
}

\section{Extended Abstract}

With the miniaturization of electronic devices, advanced cooling with super high heat flux has become the research focus in the latest decades. Traditional cooling technologies such as cooling fins and water cooling are not sufficient to handle high flux heat transfer. Amongst the various technologies, heat pipe cooling can provide the highest cooling capacity due to the liquid-vapour phase change heat transfer of a working fluid in a vacuum environment. The boiling heat transfer can be readily featured as per the well-known Nukiyama boiling curve, by which the nucleate boiling heat transfer (NBHT) is regarded as the most effective heat transfer region on a given surface. However, beyond the NBHT region, the critical heat flux (CHF) phenomenon occurs, leading to a dramatic decrease in the heat transfer ability thereby a significant rise in the surface temperature. Recently, efforts have been put in boiling enhancement on modified surfaces fabricated with micro/ nanostructures. In this research, an nano-coated heat pipe plate based on micro/nano scale heat transfer and fluid flow which is ultra-thin and flexible to be implemented is massively fabricated for breaking the restraints of conventional technologies $\left(<1 \sim 2 \mathrm{MW} / \mathrm{m}^{2}\right)$ towards a super high heat flux transportation at the magnitude of $10 \mathrm{MW} / \mathrm{m}^{2}$ that is substantially demanded in practical engineering areas.

We will present a nano-coated heat pipe plate as the advanced thermal management system using micro porous wicking flows and nano-coated porous materials to achieve high efficient heat transfer. The system consists of microchannels and porous metal foams sandwiched inside the metal casing and uses liquid-vapour two phase flows of DI water for heat transportation. A copper foam with a porosity of higher than $90 \%$ is used as the wicking material to enable the circulation of working fluid inside the device. The wicks was chemically treated to form nano layers of $\mathrm{CuO}$ on the surface of the structures which can dramatically improve the wicking performance and therefore the thermal performance of the device [1]. To optimize the effectiveness, the wicks was also treated through ultrasonic washing and acid washing except for the blackening process, which turned the surface to be super hydrophilic with a zero contact angle [2]. The whole internal micro flow system works passively without the consumption of external pumping power. Wicking and cooling performance of the device was examined in various conditions. The results showed that the wicking height of the device is much higher than the best results shown in the open literatures [3]. Furthermore, the cooling heat transfer measured in water bath experiments indicated a superior heat transportation capacity of the device, which is approximately 5 times that of traditional heat pipes and 10 times that of a copper plate. Due to the high cooling flux provided by the device, it is promising to be used for cooling of miniaturized electronics and nuclear power plant and other industrial processes where super high cooling flux is required.

\section{References}

[1] Y. Zhang and Y. P. Du, C. Shum, B. Y. Cai, N. C. H. Le, X. Chen, B. Duck, C. Fell, Y. Zhu, M. Gu, "Efficiently-cooled plasmonic amorphous silicon solar cells integrated with a nano-coated heat-pipe plate," Sci. Rep., vol.6, $24972,2016$.

[2] K. L. Chavez and D. W. Hess, "A Novel Method of Etching Copper Oxide Using Acetic Acid," J. Electrochem. Soc., vol. 148, no. 11, G640-G643, 2001. 
[3] N. Fries and M. Dreyer, "An analytic solution of capillary rise restrained by gravity," J. COLLOID INTERF. SCI., vol. 320, pp. 259-263, 2008. 